\title{
OSPF-TE Extensions for Green Routing in Optical Networks
}

Wang, Jiayuan; Ricciardi, S. ; Fagertun, Anna Manolova; Ruepp, Sarah Renée; Careglio, D. ; Dittmann, Lars

Published in:

Proceedings of the 17th OptoElectronics and Communications Conference

Publication date:

2012

Link back to DTU Orbit

Citation (APA):

Wang, J., Ricciardi, S., Fagertun, A. M., Ruepp, S. R., Careglio, D., \& Dittmann, L. (2012). OSPF-TE Extensions for Green Routing in Optical Networks. In Proceedings of the 17th OptoElectronics and Communications Conference IEEE.

\section{General rights}

Copyright and moral rights for the publications made accessible in the public portal are retained by the authors and/or other copyright owners and it is a condition of accessing publications that users recognise and abide by the legal requirements associated with these rights.

- Users may download and print one copy of any publication from the public portal for the purpose of private study or research.

- You may not further distribute the material or use it for any profit-making activity or commercial gain

- You may freely distribute the URL identifying the publication in the public portal

If you believe that this document breaches copyright please contact us providing details, and we will remove access to the work immediately and investigate your claim. 


\title{
OSPF-TE Extensions for Green Routing in Optical Networks
}

\author{
J.Wang*, S. Ricciardi**, A.V. Manolova*, S. Ruepp*, D. Careglio**, and L. Dittmann* \\ * Technical University of Denmark, Ørsteds Pl., 2800 Kgs. Lyngby, Denmark \\ ** Technical University of Catalonia, C. Jordi Girona 1-3, 08034, Barcelona, Spain
}

\begin{abstract}
This paper proposes extensions to the OSPF-TE protocol to enable green routing in GMPLS-controlled optical networks. Simulation results show a remarkable reduction in $\mathrm{CO}_{2}$ emissions by preferring network elements powered by green energy sources in the connection routing.
\end{abstract}

\section{INTRODUCTION}

The growing energy consumption and emissions of $\mathrm{CO}_{2}$ in the networks are becoming major issues for network development. This paper focuses on the network operation level, utilizing Generalized Multiprotocol Label Switching (GMPLS) control plane in optical core networks. Smart Grid power distribution networks will enable telecommunication networks to be aware of the energy source which is currently powering them. The availability of green energy sources (which emit little or no $\mathrm{CO}_{2}$ ) can be greatly influenced by natural phenomena, such as sunlight, wind and tide, or by geographic location, like hydro-electrical and geothermal power plants. Thus, in order to operate the network in a greener manner, it is necessary to have the energy sources information spread and updated in the whole network area, which can influence the routing decision process. In this paper, an extension is proposed for the Open Shortest Path First - Traffic Engineering (OSPF-TE) [1] protocol, and the impact of energy sources information on the network $\mathrm{CO}_{2}$ emissions is discussed. Simulations are conducted in a dynamic network environment, and analysis of network performance is presented, where the green energy source availability changes in time.

\section{Cost Function}

The Smart Grid provides the control plane with the energy source information, each with its own carbon footprint. All the traffic is assumed to be optical and unsplittable, and no wavelength conversion or regeneration is considered. The network is modeled as an undirected graph $G=(V, E)$, with the nodes $V$ being the optical cross-connect (OXC) and the edges $E$ being the optical links. For generality sake, we assume that devices in the network can be powered by any type of energy source based on a random selection, and the energy sources availability randomly changes on prefixed time basis. In the proposed model, OXCs, optical links and

This work was supported in part by the COST Action IC0804, the Spanish Ministry of Science and Innovation under the DOMINO project (TEC2010-18522), the Catalan Government under the contract SGR 1140 and the DIUE/ESF under the grant FI-201000740. The authors also acknowledge the support received from OPNET Technologies Inc. optical amplifiers (OAs) influence the routing decision. The proposed energy-efficient (EE) routing algorithm is based on the Dijkstra shortest path. Therefore, the cost of edge $(u, v) \in E$, used in the path computation, depend on the mentioned energy values:

$$
e_{u v}=\frac{e_{u}}{n_{u}}+\frac{e_{v}}{n_{v}}+\sum_{i=1}^{\left\lfloor l_{u v} / \Lambda\right\rfloor} e_{i}^{(u, v)},
$$

where $u, v \in V, e_{u}$ and $e_{i}^{(u, v)}$ are respectively the energy sources (Table 1) of node $u$ and of the $i^{\text {th }}$ OA on link $(u, v)$ (if any), $l_{u v}$ is the physical length of link $(u, v)$ and $\Lambda$ is the maximum link length without optical amplification. Note that the energy source contribution of nodes is divided among the number of links, indicated by $n_{u}$ and $n_{v}$.

Thus, the cost of a lightpath $P$ from source node $s$ to destination node $d$ is given by the sum of the energy costs of all the links in $P$ :

$$
E_{s d}^{P}=\sum_{(u, v) \in P} e_{u v} .
$$

In this way, each connection request will be routed selecting the less costly lightpath, lowering the carbon footprint of the network.

\section{GMPLS EXTENSIONS}

In order to obtain the energy information specified in the cost function (1), extensions to OSPF-TE protocol are proposed. Opaque Link State Advertisements (LSAs) [2] of the OSPF protocol are used to implement the proposed extensions. Considering an agile implementation, new TLVs can be added directly to the TE extensions for OSPF (TE-LSA, Opaque Type 1). The TE-LSA formats start with the standard LSA header as shown in [2]. The proposal uses Type $10 \mathrm{LSAs}$, which have an area flooding scope. TLVs are contained in the payload of LSAs. TE LSAs define two types of top-level TLVs. In this paper, Link level TLV is used as the top-level TLV. The TLV format is shown in [2]. Thus, the defined parameters are carried by setting up new sub-TLVs inside Link TLV (type 2) of TE LSAs [1]. Type in sub-TLVs is considered in the range of 32768-32777, as defined for experimental use. Table 1 lists the implementation of TLVs proposed in this paper. Each value in the TLV corresponds to a different type of energy source, where greater values correspond to higher $\mathrm{CO}_{2}$ emissions (i.e. higher energy level). Length indicates the standard length of the Value field. The proposed TE LSAs will be flooded over the whole network on a fixed time-basis, informing the current energy source information per edge. 
TABLE I. SUB-TLVS FOR TE-LSA

\begin{tabular}{|c|c|c|}
\hline Type & Length & Value \\
\hline 32768 & 4 octets & 0 (Solar, Wind, Hydro-electrical) \\
\hline 32768 & 4 octets & 1 (Nuclear) \\
\hline 32768 & 4 octets & 2 (Geothermal) \\
\hline 32768 & 4 octets & (Natural Gas) \\
\hline 32768 & 4 octets & (Biomasses) \\
\hline 32768 & 4 octets & 5 (Fuel) \\
\hline 32768 & 4 octets & 6 (Coal) \\
\hline
\end{tabular}

\section{RESULTS AND DISCUSSIONS}

The cost function and the proposed OSPF-TE extension are implemented in the event driven simulator OPNET [3]. The simulated network is the COST266 [4], with 37 nodes and 55 bidirectional links, each with sixtyfour wavelengths. More details regarding model implementation can be found in [5]. Optical amplifiers are placed every $80 \mathrm{~km}$ on each link. Label Switched Path (LSP) connection requests are generated as a Poisson process, with exponentially distributed connection duration. The total traffic load is dynamically maintained at 12 Erlangs per node. In the simulation model, first fit algorithm is applied for the wavelength assignment. At fixed time intervals (PowerChangeInterval) energy sources randomly change and a new update of the energy source information (TE-LSA) is originated and flooded between neighbors using the proposed OSPF-TE extension, and the new energy cost is updated in nodes LSA databases, and thus, used for routing calculation.

Three performance metrics have been evaluated: Energy cost (Fig. 1), average path length (in hop count) (Fig. 2) and connection blocking rate (Fig. 3). As a comparison, results using a hop based shortest path (SP) algorithm are presented. The mean value of connection duration is 6 hours, with mean inter-arrival rate of 30 minutes. PowerChangeInterval values vary among 3, 6 , 12, 24 hours, during 30 days of simulation runtime.

With the proposed algorithm, a network convergence time of $17 \mathrm{~ms}$ can be obtained, assuming a processing delay of $1 \mathrm{~ms}$ in each node. The network energy level (carbon footprint) notably decreased with the EE algorithm (regardless of the PowerChangeInterval). Compared to the SP algorithm, the hop count number has a marginal increase (less than 1 hop), indicating that using the proposed algorithm will result in slightly longer routes. An interesting result is that $\mathrm{EE}$ connection blocking rate is higher, due to the congestion on greener paths. Further simulations found that in order to reach the same blocking rate as the SP algorithm, 90 wavelengths per link have to be used, instead of 64. This implies a trade-off between the reduction of $\mathrm{CO}_{2}$ emissions and the network capacity.

\section{CONCLUSIONS}

In this paper, a green routing algorithm is proposed, aiming to route the traffic towards green energy sources. Results show a $16 \%$ reduction in the energy level and an increase of $3.3 \%$ at most in the blocking rate. Although the blocking rate can be reduced at the expenses of extra capacity, the trade-off between resource usage and energy level will be further investigated as future work.

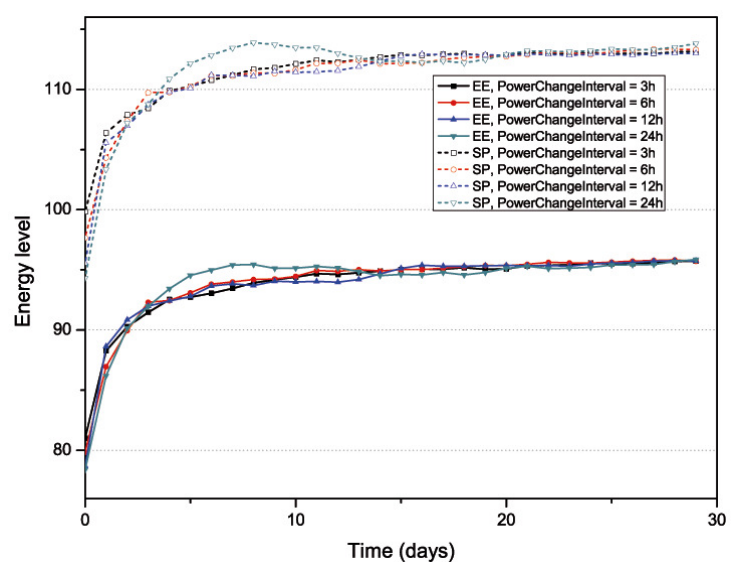

Fig. 1. Energy level ( $\mathrm{CO}_{2}$ emission units) vs Time.

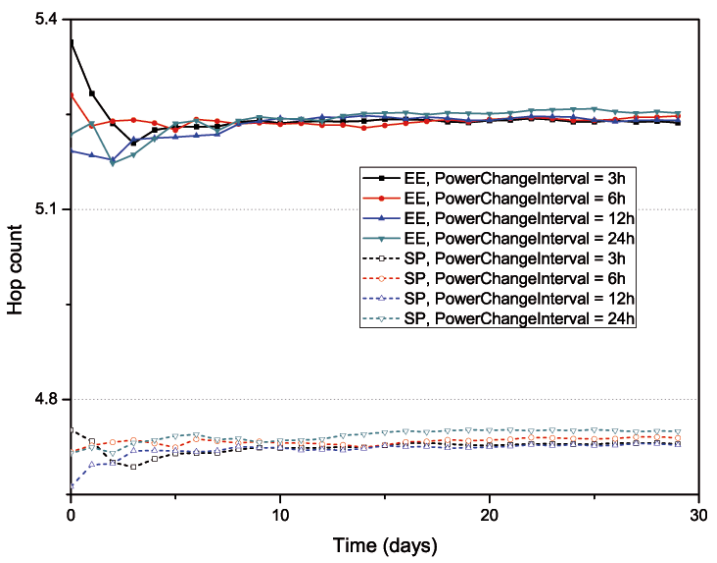

Fig. 2. Hop count $v s$ Time.

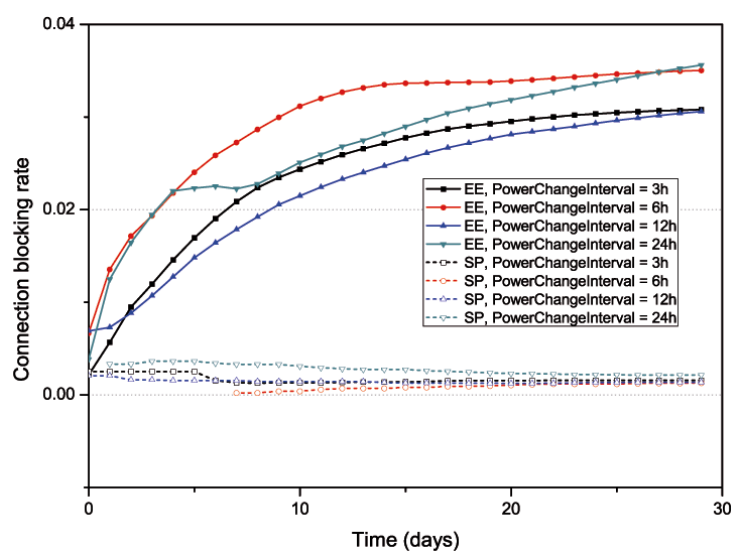

Fig. 3. Connection blocking rate vs Time.

\section{REFERENCES}

[1] D. Katz, "Traffic Engineering (TE) Extensions to OSPF Version 2," RFC 2370, Sep., 2003.

[2] R. Coltun, "The OSPF Opaque LSA Option," RFC 2328, Jul., 1998.

[3] OPNET Technologies, Inc., http://www.opnet.com

[4] R. Inkret et al., "HWDM Network Availability Performance Analysis for the COST 266 Case Study Topologies," available on the internet, 2003.

[5] J. Wang et al., "Green-Aware Routing in GMPLS Networks," ICNC workshop, Jan., 2012. 